Supporting Information

\title{
Intracellular Delivery of Recombinant RUNX2 Facilitated by Cell-Penetrating Protein for the Osteogenic Differentiation of hMSCs
}

Haein Lee ${ }^{1, \dagger}$, Seung Hyun L. Kim ${ }^{2, \dagger}$, Hyungro Yoon $^{2}$, Jina Ryu $^{2}$, Hee Ho Park
, Nathaniel S.
Hwang

${ }^{1}$ School of Chemical and Biological Engineering, Institute of Chemical Processes, Seoul National University, Seoul, Republic of Korea

${ }^{2}$ Interdisciplinary Program in Bioengineering, Seoul National University, Seoul, Republic of Korea

${ }^{3}$ Department of Biotechnology and Bioengineering, Kangwon National University, Chuncheon, Republic of Korea

${ }^{4}$ BioMax/N-Bio Institute, Institute of Bioengineering, Seoul National University, Seoul, R epublic of Korea

†These authors contributed equally to this work

*corresponding authors

nshwang@snu.ac.kr

thpark@snu.ac.kr 


\section{RUNX2}

His tag

MASNSLFSTVTPCQQNFFWDPSTSRRFSPPSSSLQPGKMSDVSPVVAAQQQQQQQQQQQQ QQQQQQQQQQQEAAAAAAAAAAAAAAAAAVPRLRPPHDNRTMVEIIADHPAELVRTDSPNFL CSVLPSHWRCNKTLPVAFKVVALGEVPDGTVVTVMAGNDENYSAELRNASAVMKNQVARFND LRFVGRSGRGKSFTLTITVFTNPPQVATYHRAIKVTVDGPREPRRHRQKLDDSKPSLFSDRLSD LGRIPHPSMRVGVPPQNPRPSLNSAPSPFNPQGQSQITDPRQAQSSPPWSYDQSYPSYLSQ MTSPSIHSTTPLSSTRGTGLPAITDVPRRISDDDTATSDFCLWPSTLSKKSQAGASELGPFSDP RQFPSISSLTESRFSNPRMHYPATFTYTPPVTSGMSLGMSATTHYHTYLPPPYPGSSQSQSGP FQTSSTPYLYYGTSSGSYQFPMVPGGDRSPSRMLPPCTTTSNGSTLLNPNLPNQNDGVDADG SHSSSPTVLNSSGRMDESVWRPYLEHHHHHH

\section{$30 \mathrm{Kc19a}$}

RUNX2

His tag

MADSDVPNDILEEQLYNSVVVADYDSAVEKSKHLYEEKKSEVITNVVNKLIRNNKMNCMEYAYQ LWLQGSKDIVRDCFPVEFRLIFAENASMASNSLFSTVTPCQQNFFWDPSTSRRFSPPSSSLQP GKMSDVSPVVAAQQQQQQQQQQQQQQQQQQQQQQQEAAAAAAAAAAAAAAAAAVPRLRP PHDNRTMVEIIADHPAELVRTDSPNFLCSVLPSHWRCNKTLPVAFKVVALGEVPDGTVVTVMA GNDENYSAELRNASAVMKNQVARFNDLRFVGRSGRGKSFTLTITVFTNPPQVATYHRAIKVTV DGPREPRRHRQKLDDSKPSLFSDRLSDLGRIPHPSMRVGVPPQNPRPSLNSAPSPFNPQGQ SQITDPRQAQSSPPWSYDQSYPSYLSQMTSPSIHSTTPLSSTRGTGLPAITDVPRRISDDDTAT SDFCLWPSTLSKKSQAGASELGPFSDPRQFPSISSLTESRFSNPRMHYPATFTYTPPVTSGMS LGMSATTHYHTYLPPPYPGSSQSQSGPFQTSSTPYLYYGTSSGSYQFPMVPGGDRSPSRMLP PCTTTSNGSTLLNPNLPNQNDGVDADGSHSSSPTVLNSSGRMDESVWRPYLEHHHHHH

\section{Supplementary Figure S1. Amino acid sequence of RUNX2 and 30Kc19 $\alpha$-RUNX2}


(A)

\section{ALP staining (Day 14)}

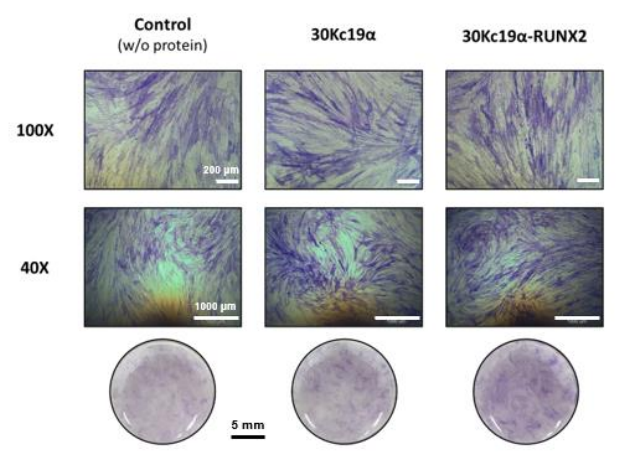

(B)

\section{ARS staining (Day 14)}

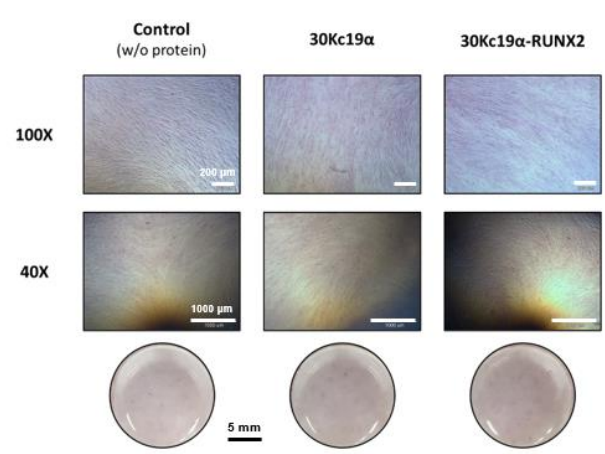

ALP staining (Day 21)

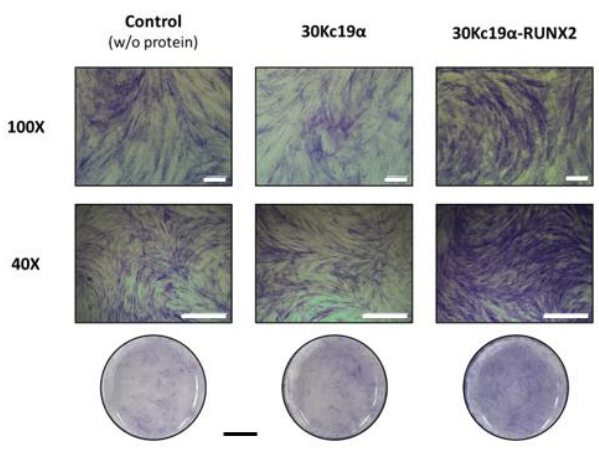

ARS staining (Day 21)

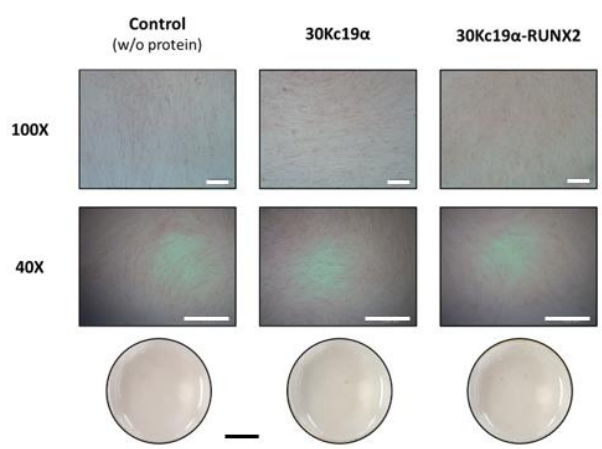

Supplementary Figure S2. ALP staining and ARS staining data when hMSCs were treated only once with $30 \mathrm{Kc19} \alpha$ and $30 \mathrm{Kc19} \alpha-\mathrm{RUNX} 2$, and cultured in OM without dexamethasone.

(A) ALP staining data of control, 30Kc19 $\alpha$, and 30Kc19 $\alpha$-RUNX2 groups on day 14 cultured in OM without dexamethasone. (B) ARS staining data of the three groups on day 21 cultured in OM without dexamethasone. hMSCs were treated only once with recombinant proteins. Scale bars: $200 \mu \mathrm{m}(100 \times$ image $), 1,000 \mu \mathrm{m}(40 \times$ image), and $5 \mathrm{~mm}$ (camera image). 